\title{
RESEARCH
}

Open Access

\section{Elucidation of SIRT-1/PGC-1a-associated mitochondrial dysfunction and autophagy in nonalcoholic fatty liver disease}

Yan Jiang ${ }^{1,2+} \mathbb{D}$, Duankai Chen ${ }^{2 \dagger}$, Qiming Gong ${ }^{2 \dagger}$, Qunqing $X u^{2}$, Dong Pan ${ }^{2}$, Feiyan Lu ${ }^{2}$ and Qianli Tang ${ }^{1,2^{*}}$

\begin{abstract}
Background: Nonalcoholic fatty liver disease (NAFLD) can lead to chronic liver diseases associated with mitochondrial damages. However, the exact mechanisms involved in the etiology of the disease are not clear.

Methods: To gain new insights, the changes affecting sirtuin 1 (SIRT-1) during liver fat accumulation was investigated in a NAFLD mouse model. In addition, the in vitro research investigated the regulation operated by SIRT-1 on mitochondrial structures, biogenesis, functions, and autophagy.

Results: In mice NAFLD, high-fat-diet (HFD) increased body weight gain, upregulated serum total cholesterol, triglycerides, aspartate aminotransferase, alanine aminotransferase, blood glucose, insulin levels, and liver malondialdehyde, and decreased liver superoxide dismutase activity. In liver, the levels of SIRT-1 and peroxisome proliferator-activated receptor-gamma coactivator -1a (PGC-1a) decreased. The expression of peroxisome proliferator-activated receptor- $a$ and Beclin-1 proteins was also reduced, while p62/SQSTM1 expression increased. These results demonstrated SIRT-1 impairment in mouse NAFLD. In a well-established NAFLD cell model, exposure of the HepG2 hepatocyte cell line to oleic acid (OA) for $48 \mathrm{~h}$ caused viability reduction, apoptosis, lipid accumulation, and reactive oxygen species production. Disturbance of SIRT-1 expression affected mitochondria. Pretreatment with Tenovin-6, a SIRT-1 inhibitor, aggravated the effect of OA on hepG2, while this effect was reversed by CAY10602, a SIRT-1 activator. Further investigation demonstrated that SIRT-1 activity was involved in mitochondrial biogenesis through PGC-1a and participated to the balance of autophagy regulatory proteins.

Conclusion: In conclusion, in high-fat conditions, SIRT-1 regulates multiple cellular properties by influencing on mitochondrial physiology and lipid autophagy via the PGC-1 a pathway. The SIRT-1/PGC-1 a pathway could be targeted to develop new NAFLD therapeutic strategies.
\end{abstract}

Keywords: Sirtuin 1, Peroxisome proliferator-activated receptor-gamma coactivator -1a, Mitochondrial physiology, Mitochondrial autophagy, Mitochondrial dysfunction; Lipid autophagy, Nonalcoholic fatty liver disease

\footnotetext{
* Correspondence: htmgx@163.com

${ }^{\dagger}$ Yan Jiang, Duankai Chen and Qiming Gong contributed equally to this work.

${ }^{1}$ Medical College of Guangxi University, Nanning 530004, Guangxi, China

${ }^{2}$ YouJiang Medical University for Nationalities, Baise 533000, Guangxi, China
}

(C) The Author(s). 2021 Open Access This article is licensed under a Creative Commons Attribution 4.0 International License, which permits use, sharing, adaptation, distribution and reproduction in any medium or format, as long as you give appropriate credit to the original author(s) and the source, provide a link to the Creative Commons licence, and indicate if changes were made. The images or other third party material in this article are included in the article's Creative Commons licence, unless indicated otherwise in a credit line to the material. If material is not included in the article's Creative Commons licence and your intended use is not permitted by statutory regulation or exceeds the permitted use, you will need to obtain permission directly from the copyright holder. To view a copy of this licence, visit http://creativecommons.org/licenses/by/4.0/. The Creative Commons Public Domain Dedication waiver (http://creativecommons.org/publicdomain/zero/1.0/) applies to the data made available in this article, unless otherwise stated in a credit line to the data. 


\section{Background}

Nonalcoholic fatty liver disease (NAFLD) is the most common chronic liver disease, with a severity ranging from simple steatosis, steatohepatitis, fibrosis, to cirrhosis, and leads to all-cause and liver-related mortality [1]. So far, the working model of NAFLD proposes the "two-hit hypothesis". Insulin resistance, which increases diet intake and hepatic lipogenesis, may cause the accumulation of triglycerides (TG) and free fatty acids in the liver. However, lipid peroxidation, mitochondrial dysfunction, and inflammation may eventually cause hepatocyte damages and liver fibrosis. Recently, some experts in the field proposed that in essence, NAFLD may be a mitochondrial disease [1].

It is well established that a high-fat diet (HFD) causes an abnormal accumulation of TG and imbalances mitochondrial function in the liver [2-5]. In progressive liver diseases, mitochondrial dysfunction produces excessive reactive oxygen species (ROS) and cytokines, which leads to hepatic inflammation and injury. Furthermore, mitochondrial dysfunction disturbs fat homeostasis in hepatic cells and eventually causes lipid accumulation, which leads to lipotoxicity [6]. It has recently been suggested that imbalanced activity of the transcription factors sterol regulatory element binding protein-1c (SREBP-1c) and peroxisome proliferator-activated receptor- $\alpha$ (PPAR- $\alpha)$ causes mitochondrial dysfunction, which in turn induces liver steatosis [7, 8]. In addition, HFD (such as palmitic acid) intake is a high-risk inducer of SREBP-1c/PPAR- $\alpha$ ratio imbalance [9].

Sirtuin 1 (SIRT-1), a master metabolic mediator, is a NAD-dependent protein deacetylase [10] involved in the control of lifespan extension and lipid metabolism, including the processes of fatty acid synthesis, oxidation, and adipocyte generation $[11,12]$. SIRT-1 activation has a beneficial effect against NAFLD through inhibiting lipogenesis, by phosphorylation of SREBP-1c [11]. Studies have demonstrated that SIRT-1 reduces oxidative stress [13, 14]. In HFD-induced obese mice, the expression of SIRT-1 is inhibited, resulting in liver metabolic damages [15]. Yet, how SIRT-1 regulates mitochondrial biology in high fatinduced livers remains poorly understood.

In the present study, the mechanisms underlying the effects of HFD on SIRT-1 in liver were investigated in vivo. The present study also tested if SIRT-1 could modulate mitochondrial physiology, and investigated the mechanisms involving SIRT-1 regulation in oleic acid (OA)-treated hepatocytes in vitro.

\section{Materials and methods Animal experiments Animal models}

Four- to five-week-old C57BL/6 mice were purchased from Changsha Tianqin Biotechnology Co., Ltd. (Changsha, China). The mice were housed in a specified constant environment and fed a normal diet for 1 week. Then, the six-week-old mice were randomly distributed between the control group (CON) and the HFD group, each group gathering eight animals. The mice were provided food and drinking water ad libitum. The mice in the CON group continued being fed an ordinary diet, while the mice in the HFD group were fed a HFD for 8 weeks. The ordinary diet was composed of (expressed as \% total calories) 65\% carbohydrates, $11 \%$ fat, $24 \%$ proteins, with a total caloric value of $3.84 \mathrm{kcal} / \mathrm{g}$, while HFD was composed of $18 \%$ carbohydrates, $62 \%$ fat, $24 \%$ proteins, with a total caloric value of $5.49 \mathrm{kcal} / \mathrm{g}$. The specific components of the HFD were diversified and were produced by Jiangsu Synergetic Pharmaceutical Bioengineering Co., Ltd. (Jiangsu, China).

\section{Collection and biochemical analysis of serum and liver tissue}

After 8 weeks of normal or HFD food intake, the 14week-old mice were fasted for $12 \mathrm{~h}$ and anesthetized using $7 \%$ chloral hydrate. Blood samples were withdrawn from the retro-orbital cavity. The serum was prepared by centrifugation at $5000 \times \mathrm{g}$ for $10 \mathrm{~min}$ at $4{ }^{\circ} \mathrm{C}$ and then stored at $-80{ }^{\circ} \mathrm{C}$ for biochemical analyses. The liver was harvested, rinsed in PBS, and wiped with filter paper. Part of the liver was fixed in formalin at $4{ }^{\circ} \mathrm{C}$, while the remaining part was stored at $-80{ }^{\circ} \mathrm{C}$ for subsequent analyses, after being quickly frozen in liquid nitrogen. The concentrations of total cholesterol (TC), TG, aspartate aminotransferase (AST), alanine aminotransferase (ALT) and blood glucose in serum were detected using biochemical reagent kits (Nanjing Jiancheng Bioengineering Institute, Nanjing, China). Biochemical assays were also performed to probe SOD activity and MAD level in liver tissues. Serum insulin was measured by the enzymelinked immunosorbent assay (ELISA) (Beyotime, Nanjing, China) methods using a TriStar LB941 system (Berthold, Wildbad, Germany).

\section{Histopathological examination}

The right lobe of the livers were collected for histopathological examination. The liver tissues fixed in formalin were cut into $5-\mu \mathrm{m}$ sections and stained with hematoxylin and eosin dyes. The livers sections frozen in liquid nitrogen were cut into $5-\mu \mathrm{m}$ using a frozen slicer (Thermo, Waltham, USA). The sections were stained with oil red $\mathrm{O}$ solution and hematoxylin. Histopathological structures were observed under a light microscope (BX53, Olympus, Tokyo, Japan).

\section{RNA extraction and analysis}

Total RNA was extracted using TRIzol reagent (CoWin Biosciences, Beijng, China) and transcribed into cDNA with a Reverse Transcription Kit (Thermo Fisher 
Scientific, Waltham, USA), according to the manufactures' instructions. Then, real-time quantitative PCR (RT-qPCR) was performed using SYBR Green Master Mix (CoWin Biosciences, Beijng, China). The reaction conditions were: pre-denaturation at $95^{\circ} \mathrm{C}$ for $10 \mathrm{~min}$, then 40 cycles of denaturation at $95^{\circ} \mathrm{C}$ for $15 \mathrm{~s}$ and annealing at $60^{\circ} \mathrm{C}$ for $30 \mathrm{~s}$. The level of GAPDH cDNA (F: 5'-CCTCGTCCCGTAGACAAAATG-3'， R: 5'-TGAG GTCAATGAAGGGGTCGT-3') was used as internal reference to quantify the level of SIRT-1 (F: 5'TTCAGAACCACCAAAGCGGA-3', R: 5' -TCCCACAG GAGACAGAAACCC-3') and PGC-1a (F: 5'-CGAGAA GCGGGAGTCTGAAAG-3', R: 5'-GAGCAGCGAA AGCGTCACA-3') cDNA. The relative mRNA expression of SIRT-1 and PGC-1a was calculated using the $2^{-\Delta \Delta C t}$ formula. All results were normalized to the GAPDH expression level.

\section{Cell experiments}

\section{Culture conditions for the HepG2 hepatocyte cell line}

The HepG2 cell line was purchased from the Cell Bank of China Academy of Sciences. Cells were maintained in Dulbecco's Modified Eagle's Medium (DMEM, $4.5 \mathrm{~g} / \mathrm{L}$ glucose, Gibco, Carlsbad, CA, USA) supplemented with $8.0 \%$ fetal bovine serum, penicillin $(100 \mathrm{U} / \mathrm{mL})$ and streptomycin $(100 \mu \mathrm{g} / \mathrm{mL})$.

\section{Cell treatment}

Cells were treated with OA (Sigma-Aldrich. St. Louis, MO, USA, catalogue \#: O1008), and SIRT-1 induction was monitored by comparing levels in unpretreated and pretreated cultures. Briefly, after inoculation, HepG2 cells were grown for $24 \mathrm{~h}$ and treated with $1.5 \mathrm{mM}$ OA for $48 \mathrm{~h}$ to induce NAFLD in cell model. The SIRT-1 inhibitor Tenovin-6, referred to as T6 (MCE, New Jersey, USA, catalogue \#: HY-15510), was used at a concentration of $2 \mu \mathrm{M}$. The SIRT-1 activator CAY-10602, referred to as CAY (MCE, New Jersey, USA, catalogue \#: HY104073), was used at a concentration of $20 \mu \mathrm{M}$. Cells were incubated with T6 or CAY for $2 \mathrm{~h}$, and subsequently stimulated with $\mathrm{OA}$ for $48 \mathrm{~h}$. The drugcontaining medium contained $12.0 \%$ of serum.

\section{Cell viability assay}

After $48 \mathrm{~h}$ of treatment, the viability of the HepG2 cells was assessed with the CCK-8 kit (ABMole, Houston, USA, catalogue \#: M4839). In brief, $10 \mu \mathrm{L}$ of CCK-8 solution was added to each well and incubated for $1 \mathrm{~h}$. The optical density (OD) was measured at $450 \mathrm{~nm}$ using the TriStar LB941 microplate reader.

\section{Oil red $O$ staining}

Oil red $\mathrm{O}$ was used to stain intracellular lipids. The cells were fixed with $10 \%$ neutral formaldehyde for $20 \mathrm{~min}$ after removing the cell culture medium. Then, the cells were stained with $5 \mathrm{mg} / \mathrm{mL}$ of oil red $\mathrm{O}$ solution for 30 $\mathrm{min}$ at room temperature. The cells were washed with PBS, and then stained with hematoxylin for $1 \mathrm{~min}$. After rinsing, the cells were observed and imaged under a light microscope (BX53, Olympus, Tokyo, Japan).

\section{Flow cytometric analysis}

The apoptosis rate in HepG2 cells was measured using the Annexin V-fluorescein Isothiocyanate/ propidium iodide (Annexin V-FITC/PI) apoptosis kit. Cells were rinsed with PBS, digested, and centrifuged. The cell density was adjusted to $1 \times 10^{6} / \mathrm{mL}$. Then, the cells were incubated with Annexin V-FITC and PI. Flow cytometric data were collected on a flow cytometer from BD Biosciences (FACS Verse, BD Biosciences, Franklin, USA) and analyzed using FlowJo software (v10.0; BD Biosciences, Franklin, USA).

\section{Immunofluorescence analysis}

Cells were seeded into glass-bottom cell culture dishes. The cells were co-transfected with Ad-GFP-LC3 and Ad-HBmTur-Mito (Hanbio, Shanghai, China, catalogue \#: HBAD-1006, 1014). Following stimulation, the localization of mitochondrial autophagy (MA) in cells was monitored under confocal laser scanning microscope (FV3000, Olympus, Tokyo, Japan). In another experiment, after removing the cell culture medium, the cells were incubated with a ROS fluorescent probe from an assay kit for $45 \mathrm{~min}$ at $37^{\circ} \mathrm{C}$. Finally, the cells were washed and analyzed with the confocal laser scanning microscope.

\section{Transmission electron microscopy analysis}

After treatment as described above, the culture medium was discarded, and $2.5 \%$ glutaraldehyde was quickly added to fix the cells. Cells were collected, centrifuged, re-fixed, dehydrated, infiltrated, and embedded. Finally, the ultrathin sections were stained with uranium and lead double solution and analyzed using a transmission electron microscope (HT7700, HITACHI, Tokyo, Japan).

\section{Western blotting analysis}

The total proteins were extracted on ice from the cells and liver tissues by ultrasonic lysis in RIPA buffer containing $1 \mathrm{mM}$ PMSF and 1\% phosphatase inhibitor cocktail. The lysates were centrifuged at $12000 \times \mathrm{g}$ for $15 \mathrm{~min}$ at $4{ }^{\circ} \mathrm{C}$. The protein concentration in the supernatant of the lysates was determined with a BCA protein quantitative kit. Next, the samples were normalized, mixed with loading buffer, and denatured by heating at $95^{\circ} \mathrm{C}$ for 5 $\mathrm{min}$. Aliquots of $50 \mathrm{mg}$ of proteins were loaded onto 8 , 10 , or $12.5 \%$ SDS-PAGE gels. The proteins were transferred onto $0.45 \mu \mathrm{m}$ PVDF membranes. The membranes were blocked and incubated overnight at $4{ }^{\circ} \mathrm{C}$ with 
primary monoclonal antibodies against SIRT-1, PGC-1 $\alpha$, Cytochrome C oxidase IV (COX-IV), PPAR-a, Mitochondrial fission factor (MFF), Mitofusin-1 (MFN1), p62/SQSTM1 (p62), Light chain 3B (LC3B), Beclin-1, and GAPDH (all from Affinity Biosciences, OH, USA). The membranes were washed with TBST and incubated with secondary antibodies at room temperature for 40 min, and after washing them five times, an enhanced chemiluminescence kit was used to detect the proteins on the membranes. The bands were quantified using the ImageJ system (v1.8.0, NIH, Bethesda, USA).

\section{Statistical analysis}

The data were analyzed using IBM SPSS statistics 23.0 software. Paired two-tailed $t$-test and one-way analysis of variance (ANOVA) were used to assess the significance of differences observed between groups. $P<0.05$ was considered statistically significant. All data are expressed as the mean \pm standard deviation $(\mathrm{M} \pm \mathrm{SD})$.

\section{Results}

HFD exacerbates obesity-related parameters in mice The mice fed HFD had a significant increase of total body weight, net weight gain and liver weight compared with the mice of the control group (Table 1A). Energy intake was increased in HFD mice compared to the controls (Table 1B), concomitantly with marked increase of

Table 1 General and hepatic parameters in control mice (CON) and high-fat-diet (HFD) mice

\begin{tabular}{|c|c|c|}
\hline & CON & HFD \\
\hline \multicolumn{3}{|c|}{ A. General and hepatic parameters } \\
\hline Initial body weight (g) & $14.49 \pm 0.55$ & $14.56 \pm 0.41$ \\
\hline Final body weight (g) & $29.38 \pm 5.58$ & $43.45 \pm 5.22^{* *}$ \\
\hline Net weight gain (g) & $14.89 \pm 5.60$ & $28.89 \pm 4.97^{* *}$ \\
\hline Liver weight (g) & $1.20 \pm 0.16$ & $2.08 \pm 0.47^{* *}$ \\
\hline \multicolumn{3}{|l|}{ B. Food and Energy intake } \\
\hline Dietary intake (g/day) & $3.64 \pm 0.37$ & $3.66 \pm 0.41$ \\
\hline Energy intake (kcal/day) & $14.64 \pm 0.68$ & $18.29 \pm 1.83^{* *}$ \\
\hline \multicolumn{3}{|l|}{ C. Serum parameters } \\
\hline $\mathrm{TC}(\mathrm{mM})$ & $1.39 \pm 0.16$ & $2.98 \pm 0.38^{* *}$ \\
\hline TG (mM) & $1.64 \pm 0.23$ & $3.95 \pm 0.54^{* *}$ \\
\hline AST (U/L) & $148.14 \pm 13.40$ & $180.24 \pm 10.08^{* *}$ \\
\hline $\operatorname{ALT}(\mathrm{U} / \mathrm{L})$ & $64.14 \pm 5.56$ & $74.84 \pm 9.05^{*}$ \\
\hline Blood glucose (mg/dL) & $122.68 \pm 12.30$ & $174.83 \pm 19.31^{* *}$ \\
\hline Insulin $(\mu \mathrm{U} / \mathrm{mL})$ & $5.90 \pm 1.95$ & $26.61 \pm 3.67^{* *}$ \\
\hline \multicolumn{3}{|l|}{ D. Enzyme activity in liver } \\
\hline MDA (nmol/g) & $37.40 \pm 7.10$ & $74.92 \pm 9.59^{* *}$ \\
\hline $\mathrm{SOD}(\mathrm{U} / \mathrm{g})$ & $78.92 \pm 7.75$ & $46.32 \pm 6.50^{* *}$ \\
\hline
\end{tabular}

Values are presented as mean \pm SD $(n=6)$. Data were analyzed by one-way analysis ANOVA. ${ }^{*},{ }^{* *}$ represents significance from the control group at $P<0.05, P<0.01$ serum TC, TG, AST, ALT, blood glucose, insulin levels and liver MDA content, and reduction of SOD activity (Table 1C-D).

HFD leads to aggregated lipids synthesis, decreased expression of SIRT-1 and PGC-1a, and alteration of autophagy-related proteins

As shown in Fig. 1, large amounts of fat forming vacuoles were observed in the livers of mice fed HFD (Fig. 1a). The oil red $\mathrm{O}$ staining also confirmed that lipid droplets accumulated in the livers of the mice from the HFD group (Fig. 1b). SIRT-1 and PGC-1 $\alpha$ expression was quantified in liver tissues by RT-qPCR and western blotting analysis. After 8 weeks of HFD regimen, liver SIRT- 1 and PGC- $1 \alpha$ expression declined (Fig. 1c-e). The expression of PPAR-a and Beclin-1 proteins also decreased, while p62 protein expression increased (Fig. 1d-e).

Effect of SIRT-1 on OA-induced cell viability in hepG2 cells To determine treatment toxicity, HepG2 cell viability was assessed by CCK-8 assay. Compared with untreated CON cultures, OA and T6 treatments decreased cell viability significantly. The combination of the two substances aggravated the cell loss. CAY had no effect on cell viability and rescued the low viability induced by OA (Fig. 2a).

\section{Effect of SIRT-1 on OA-induced cell apoptosis in hepG2 cells}

Cell death includes apoptosis and necrosis. The apoptosis provoked by the different treatments of HepG2 cells was assessed by flow cytometry. Upon OA and T6 treatments, apoptosis increased and was even more drastic in the T6-pretreated T6+OA cultures. CAY reduced cell apoptosis and rescued OA-induced apoptosis (Fig. 2b-c).

\section{Effect of SIRT-1 on OA-induced intracellular lipid in hepG2 cells}

The level of intracellular lipids was revealed by oil red $\mathrm{O}$ staining. OA or T6 treatment led to lipid accumulation manifesting as intracellular red oil droplets. This phenomenon was more pronounced with the combination of OA and T6. CAY treatment attenuated the lipid accumulation induced by OA. The oil droplets were not clearly visible in the CON and CAY groups (Fig. 3a).

\section{Effect of SIRT-1 on OA-induced ROS in hepG2 cells}

For the evaluation of mitochondrial damage, intracellular ROS was visualized by fluorescence. Mitochondrial ROS was significantly increased by $\mathrm{OA}$ and $\mathrm{T} 6$, and further enhanced by a combined treatment. In contrast, CAY did not impacted ROS whereas it alleviated OA-induced ROS production (Fig. 3b). 


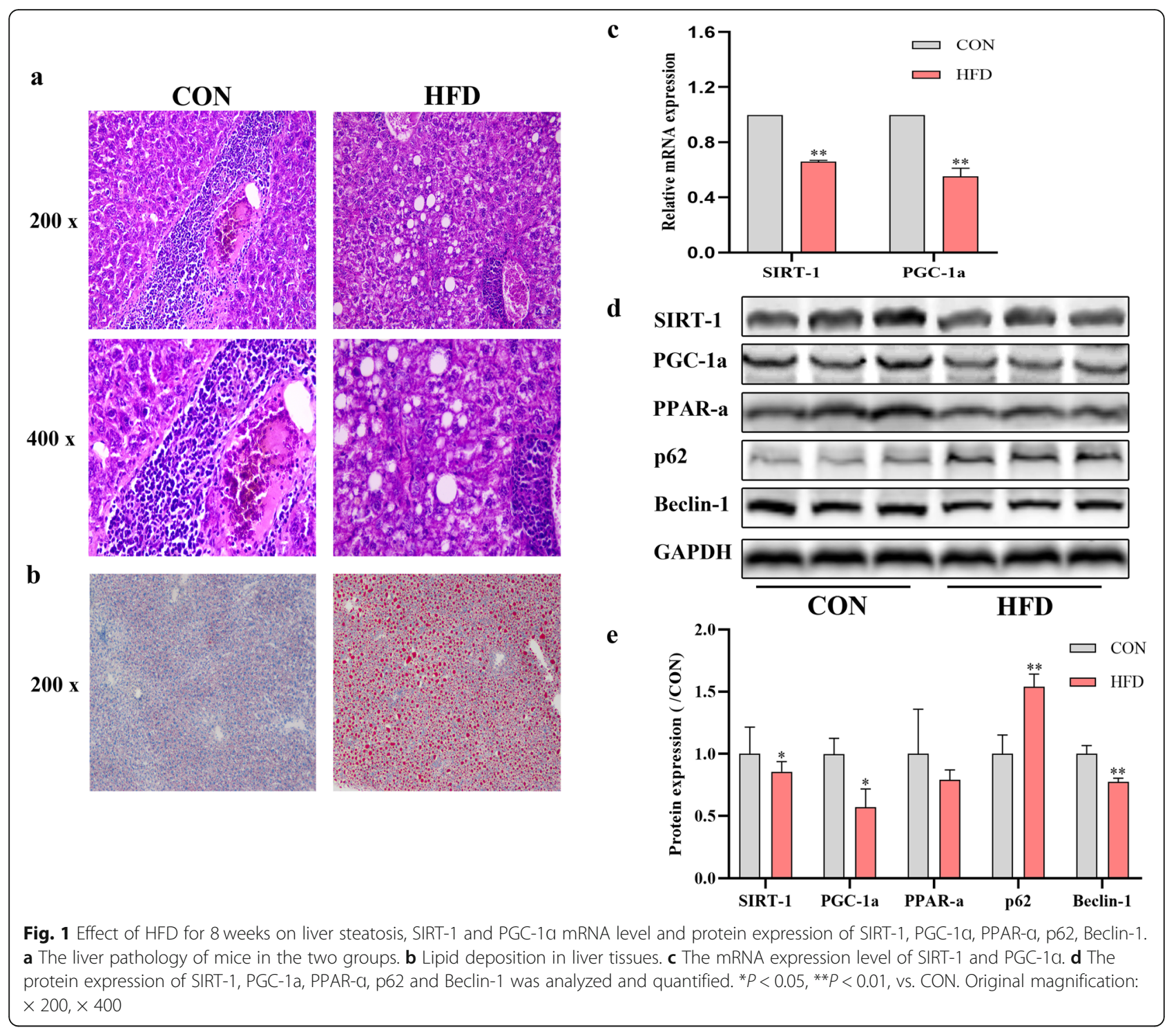

Effect of SIRT-1 on OA-induced mitochondrial autophagy in hepG2 cells

Mitochondrial autophagy was identified by staining for the autophagy protein LC3 (in green, Fig. 4a) and mitochondria (in red, Fig. 4a). Mitochondrial autophagy (in yellow/ orange, Fig. 4a) was detected in OA, CAY, T6 + OA, and CAY + OA treated cells using the confocal laser scanning microscope. T6 pretreatment severely aggravated the increase of mitochondrial autophagy provoked by OA, while CAY pretreatment appropriately increased the effect of OA (Fig. 4a).

SIRT-1 mediated the effects of OA on the substructures of hepG2 cells

HepG2 hepatocyte substructures were analyzed by transmission electron microscopy. At different stages and according to membrane enclosing cytoplasm or damaged organelles, double-membrane structures represent autophagosomes, and single-membrane structures are autolysosomes [16].

As it can be seen from the micrographs, OA- and T6treated cells have high numbers of vacuole (va), lipid drops (LD), swollen and ruptured mitochondria (SM) and autolysosomes (ASS). Lipid drops are surrounded by autolysosomes and undergo lipophagy (LA, Fig. 4b). Several autolysosomes are observed with the highest numbers being in $\mathrm{T} 6+\mathrm{OA}$ cells. Moreover, these structures are more frequent in T6treated cells, compared to other cultures (Fig. 4b).

Western blotting analysis showed that OA decreased Beclin-1 protein expression to levels similar to T6. CAY treatment reversed OA effect. Meanwhile, other autophagy-related protein markers were analyzed in the cells receiving different treatments. The level of p62 was increased in the cells receiving the OA or T6 different 


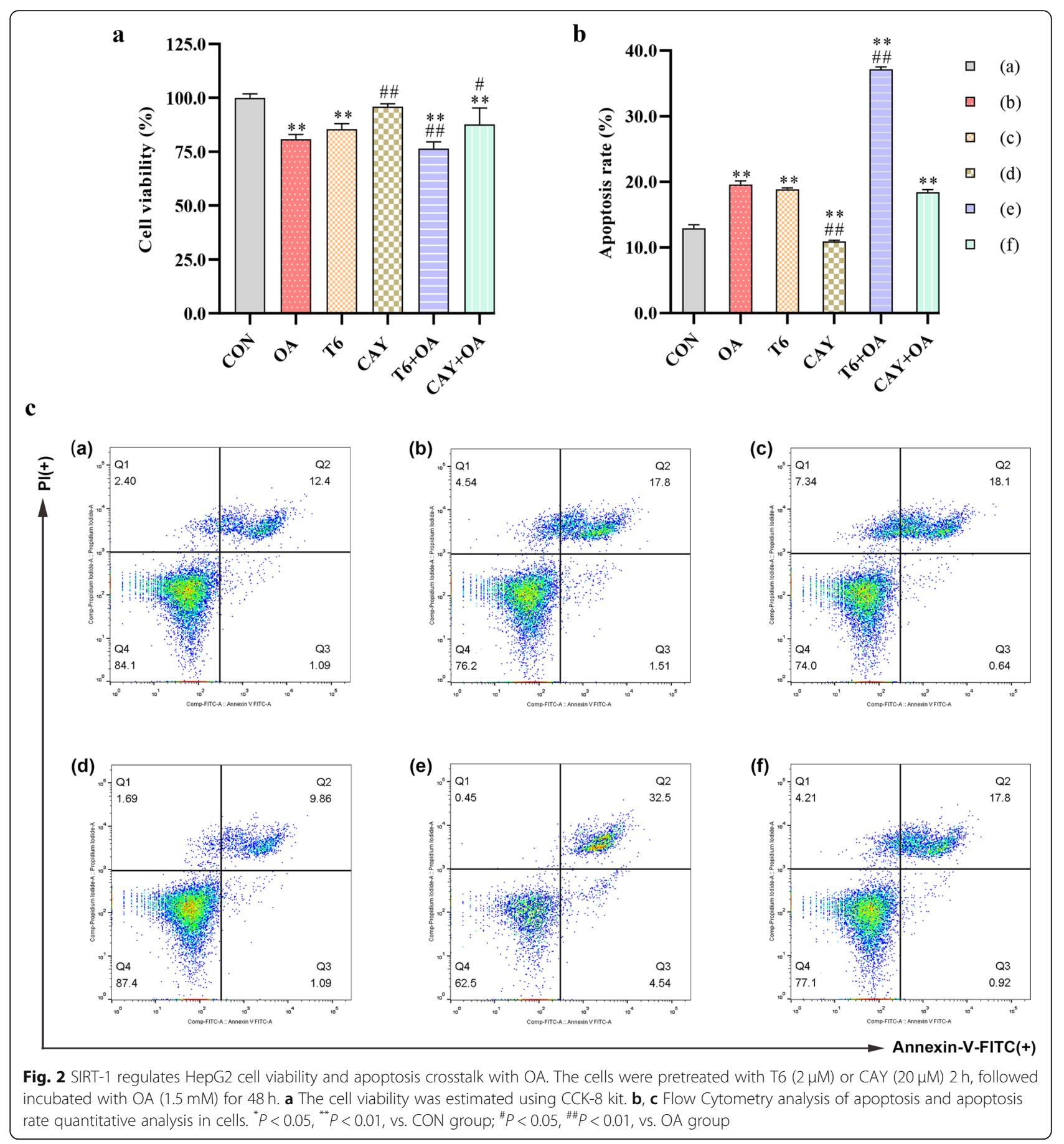

treatments but was the highest in $\mathrm{T} 6+\mathrm{OA}$ treatmed cells. Furthermore, LC3B protein level increased abnormally after stimulated by CAY or T6 pretreatment in the OAinduced cells (Fig. 5a).

\section{Effect of SIRT-1 induction upon OA treatment on hepG2 cells mitochondrial biogenesis}

To assess mitochondrial biogenesis, the effects of SIRT inhibitor and activator on mitochondrial protein expression were verified (Fig. 5b). Besides, the protein PGC- $1 \alpha$ was quantified as measurement of mitochondrial biogenesis. The results showed that PGC-1 $\alpha$ level decreased significantly after OA, T6 and combined treatment. The SIRT-1 activator CAY significantly promoted PGC- $1 \alpha$ expression compared to OA and T6. To further monitor the changes in mitochondrial biogenesis, the expressions of specific mitochondrial regulatory proteins was quantified by western blotting analysis. The results indicated that $\mathrm{OA}$ 
a

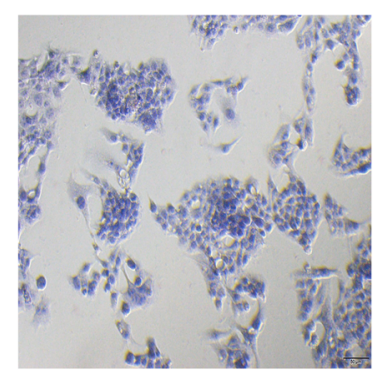

CAY

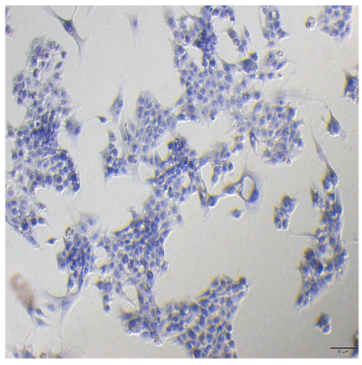

b

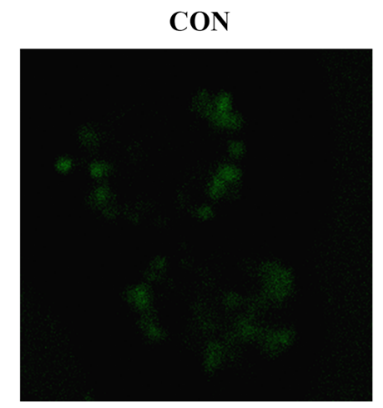

CAY

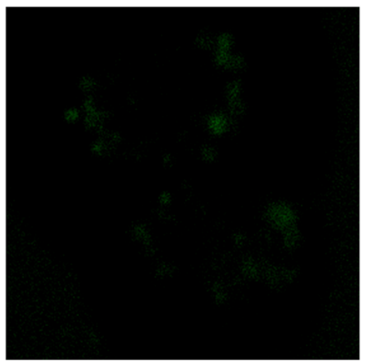

OA

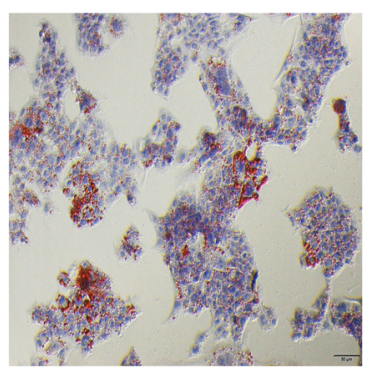

T6+OA

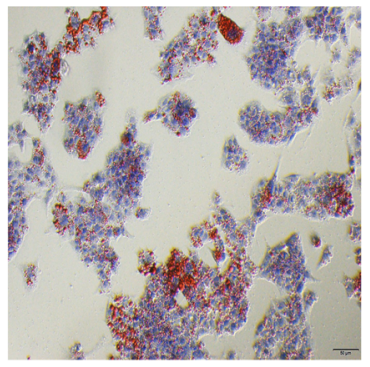

OA

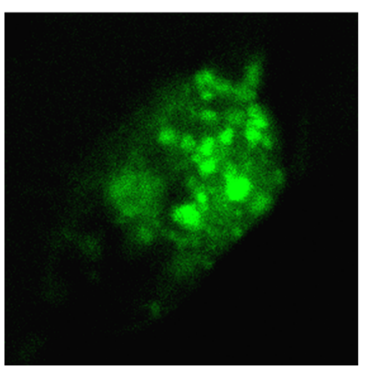

T6+OA

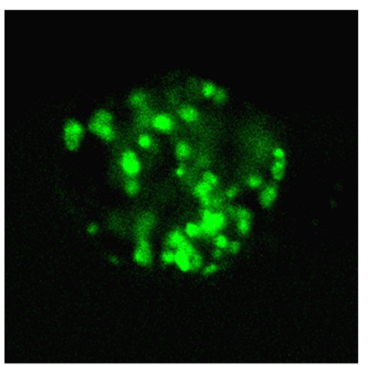

T6

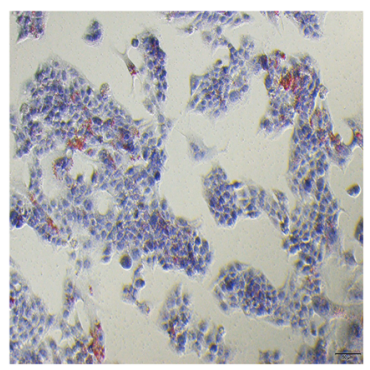

$\mathbf{C A Y}+\mathbf{O A}$

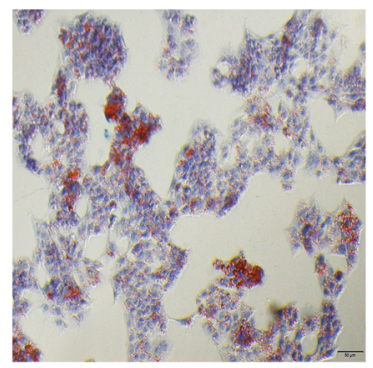

T6

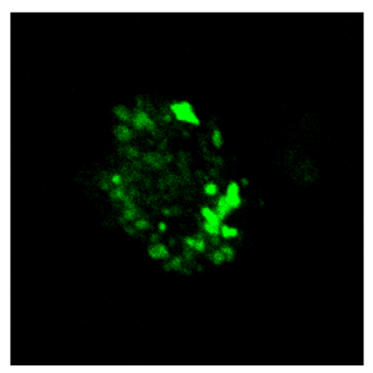

$\mathbf{C A Y}+\mathbf{O A}$

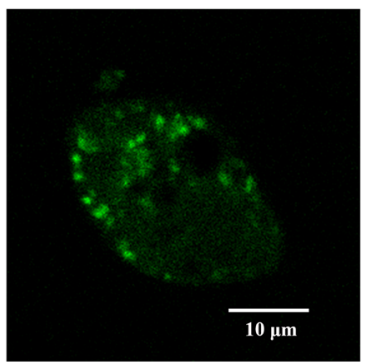

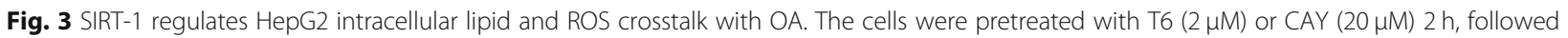
incubated with OA $(1.5 \mathrm{mM})$ for $48 \mathrm{~h}$. a The cells were stained oil red O. b Immunofluorescence detected intracellular ROS. Scale bars are $50 \mu \mathrm{m}$ and $10 \mu \mathrm{m}$ respectively

induced the mitochondrial fusion protein MFN1 but inhibited the expression of the fission protein MFF. T6 inhibited MFF protein. However, CAY promoted both mitochondrial fusion and fission, alleviated OA-promoted fusion, and rescued the inhibition operated by $\mathrm{OA}$ on mitochondrial fission. T6 pretreatment inhibited OAinduced mitochondrial fusion, while increasing OA- induced fission. Moreover, CAY and CAY + OA upregulated the expression of the mitochondrial respiratory protein COX-IV (Fig. 5b).

\section{Discussion}

SIRT-1 is a sensor of cell metabolic reactions such as stress, starvation, and caloric restriction. Target genes of 


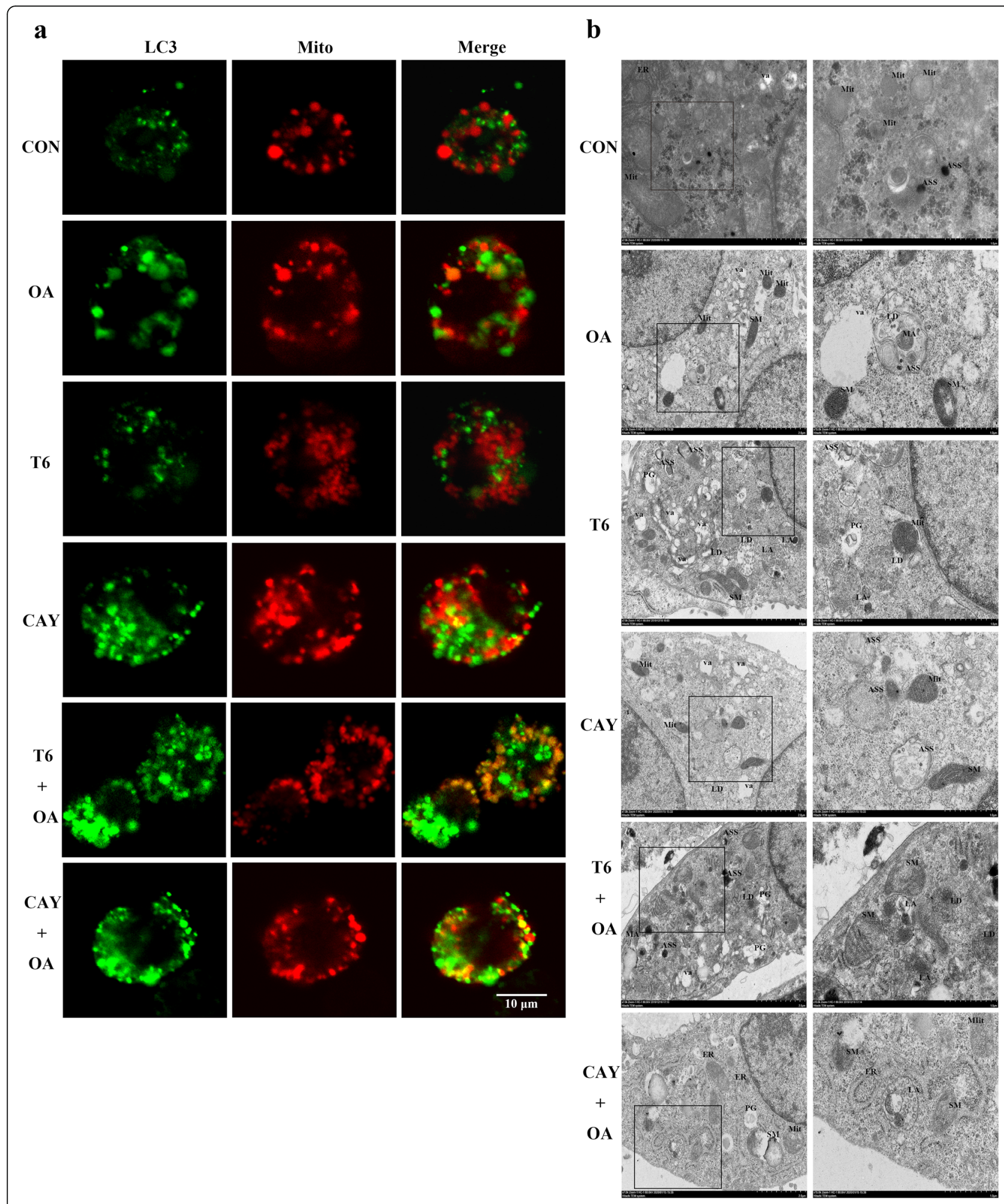

Fig. 4 SIRT-1 involved in regulation of mitochondrial autophagy and lipophages in HepG2 cells. The cells were pretreated with T6 ( $2 \mu M)$ or CAY $(20 \mu \mathrm{M}) 2 \mathrm{~h}$, followed incubated with OA $(1.5 \mathrm{mM})$ for $48 \mathrm{~h}$. a Mitochondrial autophagosome was determined. Red: mitochondria; green: LC3; yellow/ orange: merge. Scale bars are $10 \mu \mathrm{m}$ respectively. b Representative electron micrographs of cells. Mit: mitochondria; SM: swollen and ruptured mitochondria; ER: endoplasmic reticulum; ASS: autophagolysosome; PG: phagophore; va: vacuole; LD: lipid drops; LA: lipid component of autolysosome. Scalar bar $=2.0 \mu \mathrm{m}$ and $1.0 \mu \mathrm{m}$ 


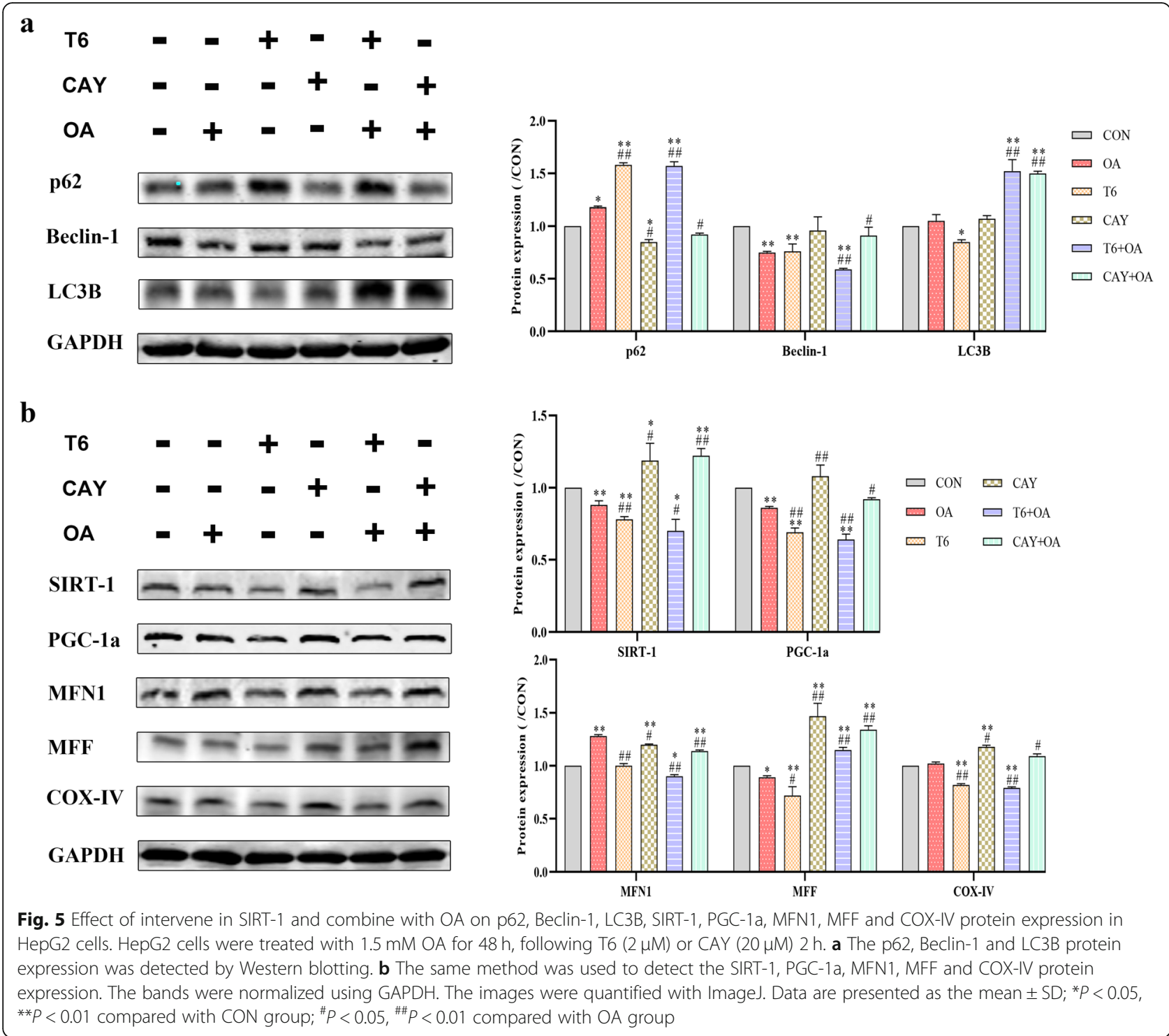

mitochondrial biogenesis can be regulated by SIRT-1 to maintain energy and metabolism stability [17]. New studies have regarded SIRT-1 as an important target for diseases associated with mitochondrial dysfunction [18]. In this experiments in mice, long-term high-fat regimen reduced the activity of SIRT-1 protein, and simultaneously, adverse changes occurred in liver structure and metabolites, such as increased levels of TG, TC, AST, ALT and MDA, and reduction of SOD activity. In addition, the interaction between SIRT-1 and autophagy responses in mitochondria under lipid accumulation process has not been explored. Further, this study deepened the insights on the effect of SIRT-1 on mitochondrial biology such as autophagy, structure, biosynthesis, and function.

Suitable oxidation of mitochondria plays a major role in energy metabolism. However, excessive ROS produced during mitochondrial dysfunction is associated with the development of NAFLD and obesity [1]. This pathological mechanism is associated with changes in NF-kB and PGC- $1 \alpha$ activities $[19,20]$. Furthermore, redundant ROS is responsible for cell apoptosis [21]. This study also proved that ROS accumulation in hepatocyte cell lines treated with OA or with a SIRT-1 inhibitor decreases cell viability and induces apoptosis. The activation of SIRT-1 prior to OA stimulation prevents this injurious effect. These results suggest that SIRT-1 is involved in the regulation of cell viability, apoptosis, and mitochondrial ROS.

Autophagy refers to the degradation of non-specific cytoplasmic proteins, organelles, and other substrates, and represents a mechanism of cell death regulation, different from apoptosis and pyroptosis. Autophagy resists 
cell pressure and promotes cell viability [22], while excessive autophagy adversely causes cell death. The gold standard markers to assess autophagy pathways are LC3B, Beclin-1, and p62. Especially, LC3B, an early marker of autophagy [20], induces autophagosome membrane expansion and fusion [22]. Besides, Beclin-1 promotes the initiation of autophagy by mediating the formation of autophagosomes. However, p62 aggregates outside the lysosomes and is involved in selective autophagy [23], in which the first steps of autophagy are impaired [24]. This knowledge was used to speculate on the trend of autophagy in cells, by detecting the changes in p62, Beclin-1, and LC3B protein expression. Experiments in mice showed that HFD increased the expression of p62 and reduced that of Beclin-1. These changes led to decreased autophagy, were not conducive to homeostatic liver lipid metabolism, and resulted in liver lipid accumulation, visible as numerous lipid droplets in liver tissues. Moreover, the same changes in p62 and Beclin-1 protein expression occurred in OA-stimulated cells. The ultrastructure analyses showed few autophagosomes. Additionally, lipid droplets were present in the intracellular space.

Moderate mitochondrial autophagy plays a crucial role in mitochondrial homeostasis by removing damaged mitochondria and contributing to cell metabolism. Abnormal mitochondria lead to imbalanced mitochondrial homeostasis and induction of mitochondrial autophagy. When mitochondria undergo autophagy, they usually show multilayered structures. In vitro, the present findings showed that excess of fat induced oil particle accumulation in the hepatocytes, and resulted in mitochondrial swelling, and even fracture. Second, the expression of the fission protein MFF diminished significantly, but that of the fusion protein MFN1 increased, both phenomena inducing abnormal mitochondrial structure. Putative mechanisms behind these changes were the increase of anomalous mitochondria to adapt to the over-accumulation of lipids. Moreover, the activation of SIRT-1 effectively promoted mitochondrial autophagy, which plays an important role in maintaining a dynamic balance in mitochondria.

PGC- $1 \alpha$ is a marker of mitochondrial biogenesis, and as a transcriptional coactivator that enhances fatty acids oxidation and glycogenesis [25], it improves intracellular energy utilization [26]. The stability of PGC-1 $\alpha$ is related to mitochondrial content $[27,28]$. The lack of PGC- $1 \alpha$ reduces the reversion of mitochondria, increases defective mitochondria, and eventually leads to cell death [29]. In addition, SIRT-1/PGC- $1 \alpha$ have been shown to be part of a network regulating mitochondrial biogenesis in skeletal muscles [30]. In this regard, the changes operated by SIRT-1 on PGC- $1 \alpha$ activity are consistent with previous reports [30, 31]. The reduction of SIRT-1 has various adverse effects on cells, such as decrease of viability, or defective mitochondrial structure and metabolism.

Mitochondrial fusion and fission regulate mitochondrial homeostasis, quantity, and even function [32]. Disrupted mitochondrial fission/fusion reduce energy production, increase oxidative stress, and even cell death [3, 32]. Mitochondrial fusion is mainly regulated by MFN1 (fusion protein, a dynamic-associated GTPase), which fuses the outer membranes of mitochondria [3]. MFF plays a role in mitochondrial fission. The decrease of SIRT-1 directly lowered mitochondrial fission by reducing MFF protein levels. This was reversed by SIRT-1 activation. OA treatment increased mitochondrial fusion, and decreased fission. Taken together, the analysis of these phenotypes indicates that mitochondria may become larger by a compensatory fusion to adapt to excessive lipid metabolism induced by OA.

It has been reported that hindered mitochondrial dynamics (fusion or fission) can lead to increased levels of some triacylglycerols, while autophagy can reverse this effect [33]. Together with this report, the present data indicate that lipid excess reduces SIRT-1, and that lipophages are not activated to a corresponding degree to degrade excessive lipids. This lack of regulation eventually leads to lipid accumulation and cell lipotoxicity.

Disruption of mitochondrial membranes, increase of ROS, fragmentation of mitochondria, and abnormal mitochondrial structure all promoted the initiation of mitochondrial autophagy [34]. The present findings demonstrate that excess of lipids and low expression of SIRT-1 lead to PGC-1 $\alpha$ imbalance, and result in disordered mitochondrial biosynthesis and excess of ROS. Ultimately, mitochondrial autophagy is initiated.

\section{Study strengths and limitations}

In the present study, there were several strengths. First, although mitochondrial disease was considered as the essence of NAFLD, few studies have performed the regulatory relationship between SIRT-1/PGC-1a and mitochondrial biology in the disease. Second, to further explore whether mitochondrial and lipid autophagy were regulated by SIRT-1, the molecular mechanism and subcellular structures influenced by SIRT-1 were included in this study. However, there are some limitations in this paper. The SIRT-1 related mechanisms were solely verified in vitro experiments. Therefore, in vivo studies are required to verify the finding.

\section{Conclusion}

In conclusion, excess of fat downregulates SIRT-1/PGC$1 \alpha$ pathway and disturbs mitochondria's biological activity, damaging them. It also decreases lipophages, causing lipid accumulation in cells and toxicity that ultimately leads to cell apoptosis. These results may point SIRT-1/ 


\section{PGC-1 $\alpha$ pathway as a good target for further exploration of adjuvant therapy in NAFLD treatment.}

\section{Abbreviations}

NAFLD: Nonalcoholic fatty liver disease; HFD: High-fat-diet; OA: Oleic acid; ROS: Reactive oxygen species; TC: Total cholesterol; TG: Triglycerides; AST: Aspartate aminotransferase; ALT: Alanine aminotransferase; MDA: Malondialdehyde; SOD: Superoxide dismutase; SIRT-1: Sirtuin 1; PGC1a: Peroxisome proliferator-activated receptor-gamma coactivator -1a; COXIV: Cytochrome c oxidase IV; p62: p62/SQSTM1, sequestosome 1; PPARa: Peroxisome proliferator-activated receptor-a; MFF: Mitochondrial fission factor; MFN1: Mitofusin-1; LC3: Light chain 3; LC3B: Light chain 3B; MA: Mitochondrial autophagy; PG: Phagophores; va: Vacuole; LD: Lipid drops; SM: Swollen and ruptured mitochondria; ASS: Autolysosomes; LA: Lipophagy; T6: Tenovin-6; CAY: CAY-10602

\section{Acknowledgements}

The authors are grateful for technical support and scientific suggestion of the researchers of science lab center at YouJiang Medical University for Nationalities

\section{Authors' contributions}

Yan Jiang, Duankai Chen and Qiming Gong conducted the experiments, and drafted the article. Qunqing Xu, Dong Pan and Feiyan Lu helped to revise the manuscript. Qianli Tang participated in its design and coordination. All authors read and approved the final manuscript.

\section{Funding}

This study was supported by Guangxi Clinical Medical Research Center of hepatobiliary Diseases (No. AD17129025), Special Funding for Guangxi Special Experts (No. GRCT[2019]13) and Guangxi Medical High-level Leading Talents Training "139" Project (No. GWKJ[2018]22).

\section{Availability of data and materials}

Not applicable.

\section{Declarations}

\section{Ethics approval and consent to participate}

The protocol was approved by the Ethics Committee of YouJiang Medical University for Nationalities (Code: 20210129001).

\section{Consent for publication}

Not applicable.

\section{Competing interests}

The authors declare that they have no competing interests.

Received: 9 January 2021 Accepted: 1 April 2021

Published online: 26 April 2021

\section{References}

1. Hu Y, Yin F, Liu Z, Xie H, Xu Y, Zhu B. Acerola polysaccharides ameliorate high-fat diet-induced non-alcoholic fatty liver disease through reduction of lipogenesis and improvement of mitochondrial functions in mice. Food Funct. 2020;11(1):1037-48. https://doi.org/10.1039/C9FO01611B.

2. Ji J, Qin Y, Ren J, Lu C, Wang R, Dai X, et al. Mitochondria-related miR-141$3 p$ contributes to mitochondrial dysfunction in HFD-induced obesity by inhibiting PTEN. Sci Rep. 2015:5:1-12.

3. Ruan XH, Ma T, Fan Y. Ablation of TMEM126B protects against heart injury via improving mitochondrial function in high fat diet (HFD)-induced mice. Biochem Biophys Res Commun. 2019;515(4):636-43. https://doi.org/10.1016/ j.bbrc.2019.05.084

4. Wohua Z, Weiming X. Weiming, Glutaredoxin 2 (GRX2) deficiency exacerbates high fat diet (HFD)-induced insulin resistance, inflammation and mitochondrial dysfunction in brain injury: a mechanism involving GSK3beta. Biomed Pharmacother. 2019;118:e108940.

5. Ferey JLA, Boudoures AL, Reid M, Drury A, Scheaffer S, Modi Z, et al. A maternal high-fat, high-sucrose diet induces transgenerational cardiac mitochondrial dysfunction independently of maternal mitochondrial inheritance. Am J Physiol Heart Circ Physiol. 2019;316:1202-10.
6. Paradies G, Paradies V, Ruggiero FM, Petrosillo G. Oxidative stress, cardiolipin and mitochondrial dysfunction in nonalcoholic fatty liver disease. World J Gastroenterol. 2014;20(39):14205-18. https://doi.org/10.3 748/wjg.v20.i39.14205.

7. Pettinelli P, Del Pozo T, Araya J, Rodrigo R, Araya AV, Smok G, et al. Enhancement in liver SREBP-1C/PPAR-alpha ratio and steatosis in obese patients: correlations with insulin resistance and $\mathrm{n}-3$ long-chain polyunsaturated fatty acid depletion. Biochim Biophys Acta. 2009;11:1080-6.

8. Valenzuela R, Videla LA. Impact of the Co-Administration of N-3 Fatty Acids and Olive Oil Components in Preclinical Nonalcoholic Fatty Liver Disease Models: A Mechanistic View. Nutrients. 2020;12(2):499.

9. Hernández-Rodas MC, Valenzuela R, Echeverría F, Rincón-Cervera MÁ, Espinosa A, Illesca P, Muñoz P, Corbari A, Romero N, Gonzalez-Mañan D, Videla LA. Supplementation with docosahexaenoic acid and extra virgin olive oil prevents liver steatosis induced by a high-fat diet in mice through PPAR- $a$ and Nrf2 upregulation with concomitant SREBP-1C and NF-kB downregulation. Mol Nutr Food Res. 2017:61(12). https://doi.org/10.1002/ mnfr.201700479.

10. Wang Y, Li X, He Z, Chen W, Lu J. Rapamycin attenuates palmitate-induced lipid aggregation by up-regulating sirt-1 signaling in AML12 hepatocytes. Pharmazie. 2016;71(12):733-7. https://doi.org/10.1691/ph.2016.6695.

11. Li J, Liu M, Yu H, Wang W, Han L, Chen Q, et al. Mangiferin improves hepatic lipid metabolism mainly through its metabolite-Norathyriol by modulating SIRT-1/AMPK/SREBP-1c signaling. Front Pharmacol. 2018;9:20114. https://doi.org/10.3389/fphar.2018.00201.

12. Khader A, Yang WL, Godwin A, Prince JM, Nicastro JM, Coppa GF, et al. Sirtuin 1 stimulation attenuates ischemic liver injury and enhances mitochondrial recovery and autophagy. Crit Care Med. 2016;44:651-63.

13. Mahmoud AR, Ali FEM, Abd-Elhamid TH, Hassanein EHM. Coenzyme Q10 protects hepatocytes from ischemia reperfusion-induced apoptosis and oxidative stress via regulation of Bax/BCl-2/PUMA and Nrf-2/FOXO-3/Sirt-1 signaling pathways. Tissue Cell. 2019;60:1-13. https://doi.org/10.1016/.tice.2019.07.007.

14. Tang Q, Len Q, Liu Z, Wang W. Overexpression of miR-22 attenuates oxidative stress injury in diabetic cardiomyopathy via Sirt 1. Cardiovasc Ther. 2018;36(2). https://doi.org/10.1111/1755-5922.12318.

15. Liou CJ, Lee YK, Ting NC, Chen YL, Shen SC, Wu SJ, et al. Wen-Chung Huang protective effects of Licochalcone a ameliorates obesity and nonalcoholic fatty liver disease via promotion of the Sirt-1/AMPK pathway in mice fed a high-fat diet. Cells. 2019;8:e447.

16. Oami T, Watanabe E, Hatano M, Teratake Y, Fujimura L, Sakamoto A, et al. Blocking liver autophagy accelerates apoptosis and mitochondrial injury in hepatocytes and reduces time to mortality in a murine Sepsis model. Shock. 2018;50(4):427-34. https://doi.org/10.1097/SHK.00000000000001040.

17. Dusabimana T, Kim SR, Kim HJ, Park SW, Kim H. Nobiletin ameliorates hepatic ischemia and reperfusion injury through the activation of SIRT-1/ FOXO3a-mediated autophagy and mitochondrial biogenesis. Exp Mol Med. 2019:51(4):1-16. https://doi.org/10.1038/s12276-019-0245-z.

18. Rigotti M, Cerbaro AF, da Silva IDR, Agostini F, Branco CS, Moura S, et al. Grape seed proanthocyanidins prevent $\mathrm{H}_{2} \mathrm{O}_{2}$-induced mitochondrial dysfunction and apoptosis via SIRT 1 activation in embryonic kidney cells. J Food Biochem. 2020;44(3):e13147. https://doi.org/10.1111/ffbc.13147.

19. Ortiz M, Soto-Alarcón SA, Orellana P, Espinosa A, Campos C, López-Arana S, et al. Suppression of high-fat diet-induced obesity-associated liver mitochondrial dysfunction by docosahexaenoic acid and hydroxytyrosol coadministration. Dig Liver Dis. 2020;52(8):895-904. https://doi.org/10.1016/j. dld.2020.04.019.

20. Echeverría F, Valenzuela R, Espinosa A, Bustamante A, Álvarez D, GonzalezMañan $\mathrm{D}$, et al. Reduction of high-fat diet-induced liver proinflammatory state by eicosapentaenoic acid plus hydroxytyrosol supplementation: involvement of resolvins RvE1/2 and RvD1/2. J Nutr Biochem. 2019:63:3543. https://doi.org/10.1016/j.jnutbio.2018.09.012.

21. Ghazipour AM, Shirpoor A, Ghiasi R, Pourheydar B, Khalaji N, Naderi R. Cyclosporine a induces testicular injury via mitochondrial apoptotic pathway by regulation of mir-34a and sirt-1 in male rats: the rescue effect of curcumin. Chem Biol Interact. 2020;327:e109180.

22. Deng JS, Jiang WP, Chen CC, Lee LY, Li PY, Huang WC, et al. Cordyceps cicadae mycelia ameliorate Cisplatin-induced acute kidney injury by suppressing the TLR4/NF-KB/MAPK and activating the HO-1/Nrf2 and Sirt-1/ AMPK pathways in mice. Oxidative Med Cell Longev. 2020;2020:e7912763.

23. Huo Y, Chen W, Zheng X, Zhao J, Zhang Q, Hou Y, et al. The protective effect of EGF-activated ROS in human corneal epithelial cells by inducing 
mitochondrial autophagy via activation TRPM2. J Cell Physiol. 2020;235(10): 7018-29. https://doi.org/10.1002/jcp.29597.

24. Carotti S, Aquilano K, Zalfa F, Ruggiero S, Valentini F, Zingariello M, et al. Lipophagy impairment is associated with disease progression in NAFLD. Front Physiol. 2020;11:850. https://doi.org/10.3389/fphys.2020.00850.

25. Keinicke H, Sun G, Mentzel CMJ, Fredholm M, John LM, Andersen B, et al. FGF21 regulates hepatic metabolic pathways to improve steatosis and inflammation. Endocr Connect. 2020;9(8):755-68. https://doi.org/10.1530/ EC-20-0152

26. Inata Y, Kikuchi S, Samraj RS, Hake PW, O'Connor M, Ledford JR, et al. Autophagy and mitochondrial biogenesis impairment contribute to agedependent liver injury in experimental sepsis: dysregulation of AMPactivated protein kinase pathway. FASEB J. 2018;32(2):728-41. https://doi. org/10.1096/fj.201700576R.

27. Miller KN, Clark JP, Anderson RM. Mitochondrial regulator PGC-1amodulating the modulator. Curr Opin Endocr Metab Res. 2019;5:37-44. https://doi.org/10.1016/j.coemr.2019.02.002.

28. Zou P, Liu L, Zheng LD, Payne KK, Manjili MH, Idowu MO, et al. Coordinated Upregulation of Mitochondrial Biogenesis and Autophagy in Breast Cancer Cells: The Role of Dynamin Related Protein-1 and Implication for Breast Cancer Treatment. Oxid Med Cell Longev. 2016;2016:4085727. https://doi. org/10.1155/2016/4085727.

29. Vainshtein A, Desjardins EM, Armani A, Sandri M, Hood DA. PGC-1a modulates denervation-induced mitophagy in skeletal muscle. Skelet Muscle. 2015;5(1):9. https://doi.org/10.1186/s13395-015-0033-y.

30. Mohamed JS, Hajira A, Pardo PS, Boriek AM. MicroRNA-149 inhibits PARP-2 and promotes mitochondrial biogenesis via SIRT-1/PGC-1a network in skeletal muscle. Diabetes. 2014;63(5):1546-59. https://doi.org/10.2337/ db13-1364.

31. Akhtar S, Siragy HM. Pro-renin receptor suppresses mitochondrial biogenesis and function via AMPK/SIRT-1/ PGC-1a pathway in diabetic kidney. PLoS One. 2019;14(12):e0225728. https://doi.org/10.1371/journal.pone.0225728

32. Li J, Zhang B, Chang X, Gan J, Li W, Niu S, et al. Silver nanoparticles modulate mitochondrial dynamics and biogenesis in HepG2 cells. Environ Pollut. 2020;256:e113430

33. Haeussler S, Köhler F, Witting M, Premm MF, Rolland SG, Fischer C, et al. Autophagy compensates for defects in mitochondrial dynamics. PLoS Genet. 2020;16(3):e1008638. https://doi.org/10.1371/journal.pgen.1008638.

34. Hasnat M, Yuan Z, Naveedb M, Khan A, Raza F, Xu D, et al. Drp1-associated mitochondrial dysfunction and mitochondrial autophagy: a novel mechanism in triptolide-induced hepatotoxicity. Cell Biol Toxicol. 2018;35: 267-80.

\section{Publisher's Note}

Springer Nature remains neutral with regard to jurisdictional claims in published maps and institutional affiliations.

Ready to submit your research? Choose BMC and benefit from:

- fast, convenient online submission

- thorough peer review by experienced researchers in your field

- rapid publication on acceptance

- support for research data, including large and complex data types

- gold Open Access which fosters wider collaboration and increased citations

- maximum visibility for your research: over $100 \mathrm{M}$ website views per year

At $\mathrm{BMC}$, research is always in progress.

Learn more biomedcentral.com/submissions 